\title{
Analytical Design of Lead-Lag Compensators on Nyquist and Nichols planes
}

\author{
R. Zanasi, ${ }^{*}$ S. Cuoghi, ${ }^{*}$ L. Ntogramatzidis ${ }^{* *}$ \\ * DII-Information Engineering Department, University of Modena and \\ Reggio Emilia, Via Vignolese 905, 41100 Modena, Italy, e-mails: \\ roberto.zanasi@unimore.it and stefania.cuoghi@unimore.it. \\ ** Department of Mathematics Statistics, Curtin University, Perth, WA, \\ Australia, e-mail: L.Ntogramatzidis@curtin.edu.au.
}

\begin{abstract}
In this paper the dynamic structure and the control properties of a new form of lead-lag compensator with complex zeros and poles are presented. A simple and exact analytical and graphical method on the Nyquist and Nichols planes for the design of lead-lag compensators satisfying design specifications on gain margin, phase margin and crossover frequency is proposed. Simulations results show the good performances of the presented method.
\end{abstract}

Keywords: Lead-Lag Compensator, Gain margin, Phase margin, Nyquist diagrams, Nichols diagrams.

\section{INTRODUCTION}

Recent works of Flores et al. (2007) and Messner (2009) show a renew interest in the design of classical controllers. A new form of lead and lag compensators with complex poles and zeros was proposed in Messner et al. (2007). Along this line, in this paper we present a new structure of lead-lag compensator with complex poles and zeros, which encompasses the classical form with real poles and zeros. The gain and phase margins (GPM) are important measures of the robustness of dynamical systems, Ho et al. (1995). Different methods can be found in the literature to satisfy GPM specifications, mostly based on trial-an-error procedures. Some of them on PID design are presented in Fung et al. (1998), Wang et al. (1999) and Lee (2004). A graphical design of lead-lag compensators on GPM specifications was presented in Yeung et al. (1998). In this paper we propose an improved graphical procedure for the design of lead-lag compensators on the Nyquist and Nichols planes. Moreover, a new numerical procedure to exactly satisfy design specifications on GPM and gain/phase crossover frequency is presented. The paper is organized as follows: in Section II the basic properties of a new form of lead-lag compensator are presented. In Section III some basic inversion formulae suitable for the design of lead-lag compensators are described. In Section IV the solutions of three different lead-lag design problems satisfying GPM specifications are presented and their graphical interpretations on Nyquist and Nichols plane are proposed. Numerical examples and conclusions end the paper.

\section{LEAD-LAG COMPENSATORS: THE GENERAL STRUCTURE}

Consider a lead-lag compensator described by the transfer function

$$
C(s)=\frac{s^{2}+2 \gamma \delta \omega_{n} s+\omega_{n}^{2}}{s^{2}+2 \delta \omega_{n} s+\omega_{n}^{2}},
$$

where $\gamma, \delta$ and $\omega_{n}$ are real and positive. When $\gamma \delta<1$ and/or $\delta<1$ the zeros and/or the poles of the lead-lag compensator
$C(s)$ are complex conjugate with negative real part. The compensator $C(s)$ is written in a general form which encompasses the classical lead-lag structure with real poles and real zeros. The compensator $C(s)$ has a unity static gain which does not change the static behavior of the controlled system. The frequency response of $C(s)$ is

$$
C(j \omega)=\frac{\omega_{n}^{2}-\omega^{2}+j 2 \gamma \delta \omega_{n} \omega}{\omega_{n}^{2}-\omega^{2}+j 2 \delta \omega_{n} \omega},
$$

which can also be written, for $\omega \neq \omega_{n}$, as

$$
C(j \omega)=\frac{1+j \gamma Y(\omega)}{1+j Y(\omega)}=\frac{1+j X(\omega)}{1+j Y(\omega)}
$$

where

$$
X(\omega)=\frac{2 \gamma \delta \omega \omega_{n}}{\omega_{n}^{2}-\omega^{2}}, \quad Y(\omega)=\frac{2 \delta \omega \omega_{n}}{\omega_{n}^{2}-\omega^{2}}, \quad \gamma=\frac{X(\omega)}{Y(\omega)} .
$$

Since it is assumed that $\gamma, \delta$ and $\omega_{n}$ are real and positive, $X(\omega)$ and $Y(\omega)$ are positive when $\omega<\omega_{n}$ and negative when $\omega>\omega_{n}$. The parameter $\gamma$ is the gain of $C(j \omega)$ at frequency $\omega=\omega_{n}$ $\left(\gamma=C\left(j \omega_{n}\right)\right)$ and is the minimum (or maximum) amplitude of $C(j \omega)$. The Nyquist diagram of $C(j \omega)$ for $\omega_{n}=1$ and for different values of parameters $\delta$ and $\gamma$ is shown in Fig. 1. The shape of these diagrams are circles, as the following property explains.

Definition 1. Let $\mathscr{C}(\gamma)$ denote the set of all the lead-lag compensators $C(s)$ as defined in (1) having the same parameter $\gamma$, that is

$$
\mathscr{C}(\gamma)=\left\{C(s) \text { as in }(1) \mid \delta>0, \omega_{n}>0\right\} .
$$

Moreover, let $\mathscr{C}_{\gamma}(s) \in \mathscr{C}(\gamma)$ denote one element of set $\mathscr{C}(\gamma)$ chosen arbitrarily.

Property 1. The shape of the frequency response $\mathscr{C}_{\gamma}(j \omega)$ of $\mathscr{C}_{\gamma}(s)$ on the Nyquist plane, see Fig. 1 , is a circle with center $C_{0}$ and radius $R_{0}$

$$
C(\gamma)=C_{0}+R_{0} e^{j \theta}, \quad C_{0}=\frac{\gamma+1}{2}, \quad R_{0}=\frac{|\gamma-1|}{2},
$$




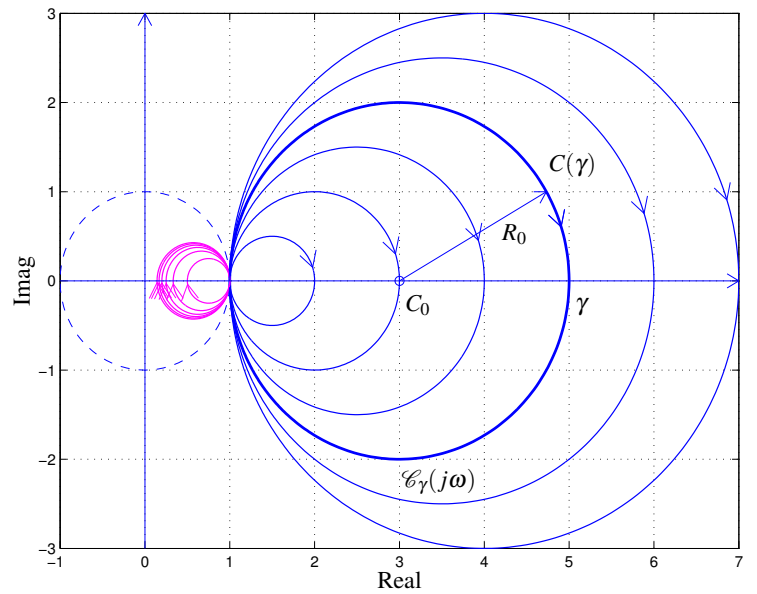

Fig. 1. Nyquist diagrams of $C(j \omega)$ when $\omega_{n}=1,(\delta=1.5$, $\gamma=[2: 1: 7]$, blue lines $)$ and $(\gamma \delta=1.5, \gamma=1 . /[2: 1: 7]$, magenta lines). The thick blue line corresponds to $\delta=1.5$ and $\gamma=5$.

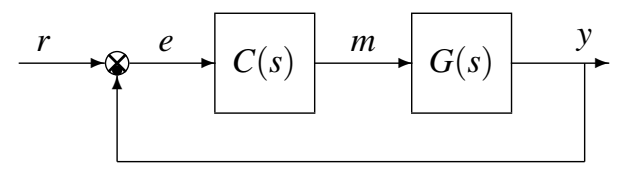

Fig. 2. Unity feedback control structure.

where $\theta \in[0,2 \pi]$. The intersections of $\mathscr{C}_{\gamma}(j \omega)$ with the real axis occur at points 1 and $\gamma$. The shape does not depend on $\delta$ and $\omega_{n}$.

Proof. One can directly verify that the distance $d=\mid \mathscr{C}_{\gamma}(j \omega)-$ $C_{0} \mid$ of the generic point $\mathscr{C}_{\gamma}(j \omega)$ from the center $C_{0}$ is constant and equal to radius $R_{0}$.

\section{LEAD-LAG COMPENSATORS $C\left(s, \omega_{n}\right)$ MOVING A POINT $A$ TO A POINT $B$}

Consider the block-diagram shown in Fig. 2, where $G(s)$ denotes the transfer function of the LTI plant to be controlled, which may include the gain and the integration terms required to meet the steady-state accuracy specifications. Let $C\left(j \omega_{0}\right)=$ $M_{0} e^{j \varphi_{0}}$ denote the value of the frequency response of the compensator $C(s)$ at frequency $\omega_{0}$, where $M_{0}=M\left(\omega_{0}\right)$ and $\varphi_{0}=$ $\varphi\left(\omega_{0}\right)$. To study how $C\left(j \omega_{0}\right)$ affects $G(j \omega)$ at frequency $\omega_{0}$, let us consider two generic points $A=M_{A} e^{j \varphi_{A}}$ and $B=M_{B} e^{j \varphi_{B}}$ of the complex plane. Referring to Fig. 3, we say that point $A$ can be moved to point $B$ if a value $C\left(j \omega_{0}\right)$ exists such that $B=C\left(j \omega_{0}\right) \cdot A$, that is if and only if the following conditions hold:

$$
M_{B}=M_{A} M_{0}, \quad \varphi_{B}=\varphi_{A}+\varphi_{0} .
$$

Definition 2. Given a point $B \in \mathbb{C}$, let us define "admissible domain of lead-lag compensator $C(s)$ for reaching point B" as

$$
\mathscr{D}_{B}^{-}=\left\{A \in \mathbb{C} \mid \exists \gamma, \delta, \omega_{n}>0, \exists \omega \geq 0: C(j \omega) \cdot A=B\right\}
$$

The domain $\mathscr{D}_{B}^{-}$on Nyquist plane is shown in gray in Fig. 3. It can be easily shown that the domain $\mathscr{D}_{B}^{-}$on Nyquist plane can always be obtained as the union of the disk $\mathscr{D}_{B 1}^{-}$having its

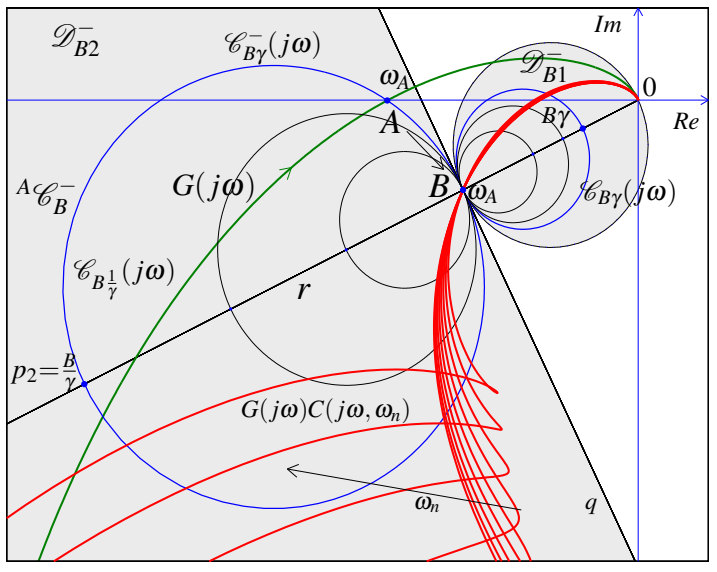

Fig. 3. Admissible domain $\mathscr{D}_{B}^{-}$and graphical design of lead-lag compensators $C\left(j \omega, \omega_{n}\right)$ moving point $A$ to $B$.

diameter on the segment $\overline{B 0}$ and the external half-plane $\mathscr{D}_{B 2}^{-}$ delimited by the straight line $q$ passing through point $B$ and perpendicular to segment $\overline{B 0}$.

Definition 3. Given a point $B \in \mathbb{C}$, let $\mathscr{C}_{B}(\gamma)$ and $\mathscr{C}_{B}^{-}(\gamma)$ denote the sets of lead-lag compensators $C(s)$ defined as follows

$$
\begin{aligned}
& \mathscr{C}_{B}(\gamma)=\{B \cdot C(s) \mid C(s) \in \mathscr{C}(\gamma)\} \\
& \mathscr{C}_{B}^{-}(\gamma)=\left\{\frac{B}{C(s)} \mid C(s) \in \mathscr{C}(\gamma)\right\}
\end{aligned}
$$

with $\mathscr{C}(\gamma)$ defined in (5). Moreover, let $\mathscr{C}_{B \gamma}(s) \in \mathscr{C}_{B}(\gamma)$ and $\mathscr{C}_{B \gamma}^{-}(s) \in \mathscr{C}_{B}^{-}(\gamma)$ denote particular elements of the two sets $\mathscr{C}_{B}(\gamma)$ and $\mathscr{C}_{B}^{-}(\gamma)$ chosen arbitrarily.

Property 2. Given $\gamma>0$, the two sets $\mathscr{C}_{B}^{-}(\gamma)$ and $\mathscr{C}_{B}\left(\frac{1}{\gamma}\right)$ coincide, i.e.,

$$
\mathscr{C}_{B}^{-}(\gamma)=\mathscr{C}_{B}\left(\frac{1}{\gamma}\right)
$$

and the Nyquist diagram of the frequency responses $\mathscr{C}_{B \gamma}^{-}(j \omega)$ and $\mathscr{C}_{B \frac{1}{\gamma}}(j \omega)$ corresponding to $\mathscr{C}_{B \gamma}^{-}(s)$ and $\mathscr{C}_{B \frac{1}{\gamma}}(s)$, respectively, have the same shape, see Fig. 3. The intersections $p_{1}$ and $p_{2}$ of $\mathscr{C}_{B \gamma}^{-}(j \omega)$ with the straight line r passing through points 0 and $B$ are $p_{1}=B$ and $p_{2}=\frac{B}{\gamma}$, respectively.

Proof. Each element $\mathscr{C}_{B \gamma}^{-}(s)$ of the set $\mathscr{C}_{B}^{-}(\gamma)$ also belongs to the set $\mathscr{C}_{B}\left(\frac{1}{\gamma}\right)$. In fact, from (5), (8) and (9) it follows that

$$
\begin{aligned}
\mathscr{C}_{B \gamma}^{-}(s) & =\left.B \frac{s^{2}+2 \delta \omega_{n} s+\omega_{n}^{2}}{s^{2}+2 \gamma \delta \omega_{n} s+\omega_{n}^{2}}\right|_{\substack{\forall \delta>0 \\
\forall \omega_{n}>0}}\left|\frac{s^{2}+2\left(\frac{1}{\gamma}\right) \bar{\delta} \omega_{n} s+\omega_{n}^{2}}{s^{2}+2 \bar{\delta} \omega_{n} s+\omega_{n}^{2}}\right|_{\substack{\forall \bar{\delta}>0 \\
\forall \omega_{n}>0}} \in \mathscr{C}_{B}\left(\frac{1}{\gamma}\right),
\end{aligned}
$$

where $\bar{\delta}=\gamma \delta$. In the same way it can be easily proved that each element $\mathscr{C}_{B \frac{1}{\gamma}}(s)$ of $\mathscr{C}_{B}\left(\frac{1}{\gamma}\right)$ also belongs to $\mathscr{C}_{B}^{-}(\gamma)$, and therefore $\mathscr{C}_{B}^{-}(\gamma)$ and $\mathscr{C}_{B}\left(\frac{1}{\gamma}\right)$ coincide. Moreover, the shape of the Nyquist diagrams of $\mathscr{C}_{B \gamma}^{-}(s)$ and $\mathscr{C}_{B \frac{1}{\gamma}}(s)$ depend only on $\gamma$ and therefore they coincide. From (6) and (10) it follows that the Nyquist diagram of $\mathscr{C}_{B \gamma}^{-}(s)$ is a circle whose center $C_{0}=B(\gamma+1) /(2 \gamma)$ lies on the straight line $r$ passing through points 0 and $B$, see 
Fig. 3, whose radius is $R_{0}=|\gamma-1| /(2 \gamma)$ and its intersections with the straight line $r$ occur at points $p_{1}=B$ and $p_{2}=\frac{B}{\gamma}$.

Definition 4. (Inversion Formulae) Given two points $A=$ $M_{A} e^{j \varphi_{A}}$ and $B=M_{B} e^{j \varphi_{B}}$ of the complex plane $\mathbb{C}$, the inversion formulae $X(A, B)$ and $Y(A, B)$ are defined as

$$
\begin{aligned}
& X(A, B)=\frac{M-\cos \varphi}{\sin \varphi}, \\
& Y(A, B)=\frac{\cos \varphi-\frac{1}{M}}{\sin \varphi},
\end{aligned}
$$

where $M=\frac{M_{B}}{M_{A}}$ and $\varphi=\varphi_{B}-\varphi_{A}$. Moreover, from (11) it follows that $\gamma(A, B)=\frac{M-\cos \varphi}{\cos \varphi-\frac{1}{M}}$.

These formulae are similar to the ones used in Phillips (1985) and are the same Inversion Formulae introduced and used in Marro and R. Zanasi (1998) and Zanasi and Morselli (2009) for the continuous-time case.

Property 3. (From $A$ to $B$ ) Given a point $B \in \mathbb{C}$ and chosen a point $A$ of the frequency response $G(j \omega)$ at frequency $\omega_{A}$ belonging to the admissible domain $\mathscr{D}_{B}^{-}$, i.e., $A=G\left(j \omega_{A}\right) \in$ $\mathscr{D}_{B}^{-}$, the set $C\left(s, \omega_{n}\right)$ of all the lead-lag compensators $C(s)$ that move point $A$ to point $B$ is obtained from (1) using the parameters

$$
\gamma=\gamma(A, B)=\frac{X(A, B)}{Y(A, B)}>0, \quad \delta=Y(A, B) \frac{\omega_{n}^{2}-\omega_{A}^{2}}{2 \omega_{n} \omega_{A}}>0
$$

for all $\omega_{n}>0$ such that $\delta>0$ and with parameters $X(A, B)$ and $Y(A, B)$ obtained using (11).

Proof. For $\omega=\omega_{A}$, relations (4) can be rewritten as

$$
\gamma=\frac{X\left(\omega_{A}\right)}{Y\left(\omega_{A}\right)}, \quad \delta=Y\left(\omega_{A}\right) \frac{\omega_{n}^{2}-\omega_{A}^{2}}{2 \omega_{n} \omega_{A}} .
$$

Substituting in (1) yields

$$
C\left(s, \omega_{n}\right)=\frac{s^{2}+X\left(\omega_{A}\right) \frac{\omega_{n}^{2}-\omega_{A}^{2}}{\omega_{A}} s+\omega_{n}^{2}}{s^{2}+Y\left(\omega_{A}\right) \frac{\omega_{n}^{2}-\omega_{A}^{2}}{\omega_{A}} s+\omega_{n}^{2}} .
$$

The frequency response of $C\left(s, \omega_{n}\right)$ at frequency $\omega_{A}$ is equal to the constant value

$$
C\left(j \omega_{A}, \omega_{n}\right)=C\left(j \omega_{A}\right)=\frac{1+j X\left(\omega_{A}\right)}{1+j Y\left(\omega_{A}\right)} .
$$

From equations $B=C\left(j \omega_{0}\right) \cdot A$ and (7) it is evident that point $A=G\left(j \omega_{A}\right)=M_{A} e^{j \varphi_{A}}$ can be moved to point $B=M_{B} e^{j \varphi_{B}}$ if and only if

$$
C\left(j \omega_{A}\right)=M e^{j \varphi}=\frac{M_{B}}{M_{A}} e^{j\left(\varphi_{B}-\varphi_{A}\right)} .
$$

Solving equations (13) and (14) with respect to $X\left(\omega_{A}\right)$ and $Y\left(\omega_{A}\right)$, one obtains the Inversion Formulae $X=X(A, B)$ and $Y=Y(A, B)$ introduced in Definition 4. The hypothesis that point $A$ belongs to the admissible domain $\mathscr{D}_{B}^{-}$ensures, see Definition 2, that there exist admissible lead-lag controllers $C\left(s, \omega_{n}\right)$ moving point $A$ to point $B$ which are characterized by positive parameters $\gamma, \delta$ and $\omega_{n}$. All the admissible values of $\omega_{n}$ are those satisfy $\delta>0$ in (12).

\section{SYNTHESIS OF LEAD-LAG COMPENSATORS}

Design Problem A: $\left(\phi_{m}, G_{m}\right)$. Given the control scheme of Fig. 2, the transfer function $G(s)$ and the design specifications

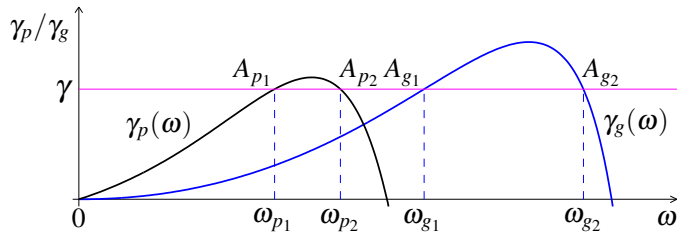

Fig. 4. Functions $\gamma_{g}(\omega)$ (blue line) and $\gamma_{p}(\omega)$ (black line).

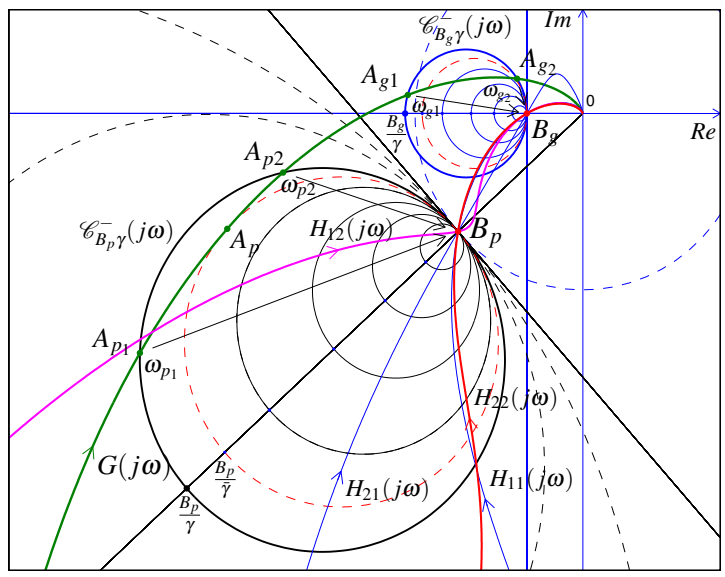

Fig. 5. Graphical solution of the DPA on the Nyquist plane.

on the phase margin $\phi_{m}$ and gain margin $G_{m}$, design a leadlag compensator $C(s)$ such that the loop gain transfer function $C(j \omega) G(j \omega)$ passes through points $B_{p}=e^{j\left(\pi+\phi_{m}\right)}$ and $B_{g}=$ $-1 / G_{m}$.

Solution A. Let $B_{p}=e^{j\left(\pi+\phi_{m}\right)}$ and $B_{g}=-1 / G_{m}=M_{B_{g}} e^{j \varphi_{B g}}$ denote the points corresponding to the desired phase margin $\phi_{m}$ and gain margin $G_{m}$. The set $C_{\gamma}\left(s, \omega_{p}, \omega_{g}\right)$ of all the compensators $C(s)$ which solve the Design Problem A (DPA) is obtained as follows:

a) find all the pairs $\left(\omega_{p}, \omega_{g}\right) \in S_{\gamma \omega}$ of frequencies which solve the equation

$$
\gamma=\gamma_{p}\left(\omega_{p}\right)=\gamma_{g}\left(\omega_{g}\right)
$$

where the gain $\gamma>0$ is chosen arbitrarily, $S_{\gamma \omega}$ is the set of all the pairs $\left(\omega_{p}, \omega_{g}\right)$ satisfying (15), and functions $\gamma_{p}\left(\omega_{p}\right)$ and $\gamma_{g}\left(\omega_{g}\right)$ are defined as

$$
\gamma_{p}\left(\omega_{p}\right)=\frac{X_{p}}{Y_{p}}, \quad \gamma_{g}\left(\omega_{g}\right)=\frac{X_{g}}{Y_{g}},
$$

where the coefficients $X_{p}=X\left(A_{p}\left(\omega_{p}\right), B_{p}\right), Y_{p}=Y\left(A_{p}\left(\omega_{p}\right), B_{p}\right)$, $X_{g}=X\left(A_{g}\left(\omega_{g}\right), B_{g}\right)$ and $Y_{g}=Y\left(A_{g}\left(\omega_{g}\right), B_{g}\right)$ are obtained using the inversion formulae (11), $A_{p}=G\left(j \omega_{p}\right)=M_{A_{p}}\left(\omega_{p}\right) e^{j \varphi_{A_{p}}\left(\omega_{p}\right)}$ and $A_{g}=G\left(j \omega_{g}\right)=M_{A_{g}}\left(\omega_{g}\right) e^{j \varphi_{A_{g}}\left(\omega_{g}\right)}$.

b) for each pair $\left(\omega_{p}, \omega_{g}\right) \in S_{\gamma \omega}$ compute

$$
\begin{gathered}
\omega_{n}=\sqrt{\frac{X_{g} \omega_{g}-X_{p} \omega_{p}}{\frac{X_{g}}{\omega_{g}}-\frac{X_{p}}{\omega_{p}}}}=\sqrt{\frac{Y_{g} \omega_{g}-Y_{p} \omega_{p}}{\frac{Y_{g}}{\omega_{g}}-\frac{Y_{p}}{\omega_{p}}}}>0, \\
\delta=Y_{p} \frac{\omega_{n}^{2}-\omega_{p}^{2}}{2 \omega_{n} \omega_{p}}=Y_{g} \frac{\omega_{n}^{2}-\omega_{g}^{2}}{2 \omega_{n} \omega_{g}}>0 .
\end{gathered}
$$

A solution $C_{\gamma}\left(s, \omega_{p}, \omega_{g}\right)$ of Design Problem $\left(\phi_{m}, G_{m}\right)$ exists only if: 1) $\gamma$ satisfies

$$
0<\gamma<\min \left[\max \left(\gamma_{p}\left(\omega_{p}\right)\right), \max \left(\gamma_{g}\left(\omega_{g}\right)\right)\right]
$$




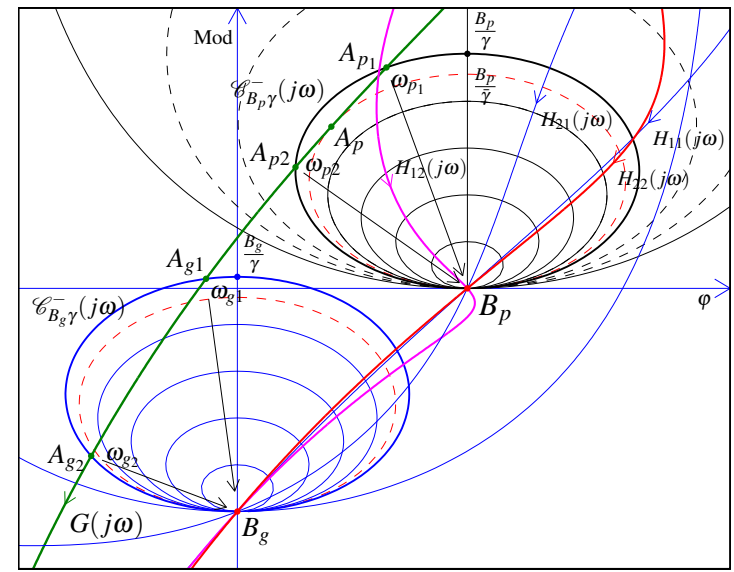

Fig. 6. Graphical solution of the DPA on the Nichols plane.

2) $S_{\gamma \omega}$ is not empty; 3) $A_{p}\left(\omega_{p}\right) \in \mathscr{D}_{B_{p}}^{-}$and $A_{g}\left(\omega_{g}\right) \in \mathscr{D}_{B_{g}}^{-}$; 4) $\omega_{n}$ in (17) and $\delta$ in (18) are real and positive.

The proof hinges on the fact that $C(s)$ has to be designed to move point $A_{p}=G\left(j \omega_{p}\right)$ to point $B_{p}$ and point $A_{g}=G\left(j \omega_{g}\right)$ to point $B_{g}$. Parameters $\gamma$ and $\delta$ are obtained as described in Property 3 for $\omega_{A}=\omega_{p}, A=A_{p}$ and $B=B_{p}$ to satisfy the first condition, and for $\omega_{A}=\omega_{g}, A=A_{g}$ and $B=B_{g}$ to satisfy the second condition.

The solution of (15) can also be obtained graphically by plotting $\gamma_{p}(\omega)$ and $\gamma_{g}(\omega)$ and by finding, for each admissible value of $\gamma$, all the pairs $\left(\omega_{p}, \omega_{g}\right) \in S_{\omega_{p}}$ where $\gamma_{p}\left(\omega_{p}\right)$ and $\gamma_{g}\left(\omega_{g}\right)$ intersect the horizontal line $\gamma$, see Fig. 4. In the example of Fig. 4 there are four different solutions: $S_{\gamma \omega}=\left\{\left(\omega_{p 1}, \omega_{g 1}\right),\left(\omega_{p 1}, \omega_{g 2}\right),\left(\omega_{p 2}, \omega_{g 1}\right),\left(\omega_{p 2}, \omega_{g 2}\right)\right\}$. The loop gain frequency responses $H_{11}(s), H_{12}(s), H_{21}(s)$ and $H_{22}(s)$ of these four solutions on the Nyquist plane are shown in Fig. 5. These solutions are acceptable only if constraints $\delta>0$ and $\omega_{n}>0$ given in (17) and (18) are satisfied.

\subsection{Graphical Solutions on Nyquist and Nichols planes.}

The graphical solution of (15) can also be obtained by using the graphical constructions shown in Fig. 5 and Fig. 6.

a) Graphical Solution on Nyquist plane. The graphical construction shown in Fig. 5 is obtained as follows:

1) given points $B_{p}$ and $B_{g}$ and a desired value for $\gamma>0$, draw the circles having their diameters on the segments $\left(B_{p}, B_{p} / \gamma\right)$ and $\left(B_{g}, B_{g} / \gamma\right)$. From Property (2) they coincide with the frequency responses $\mathscr{C}_{B_{p} \gamma}^{-}(j \omega)$ and $\mathscr{C}_{B_{g} \gamma}^{-}(j \omega)$.

2) if the frequency response $G(j \omega)$ does not intersect both circles $\mathscr{C}_{B_{p} \gamma}^{-}(j \omega)$ and $\mathscr{C}_{B_{g} \gamma}^{-}(j \omega)$, the chosen value of $\gamma$ is not acceptable.

3) otherwise, each pair $\left(\omega_{p}, \omega_{g}\right)$ corresponding to the intersections of $G(j \omega)$ with circles $\mathscr{C}_{B_{p} \gamma}^{-}(j \omega)$ and $\mathscr{C}_{B_{g} \gamma}^{-}(j \omega)$ is a possibile solution for Design Problem A $\left(\phi_{m}, G_{m}\right)$.

This graphical solution hinges on the fact that frequencies $\omega_{p}$ and $\omega_{g}$ satisfy (15) only if $G\left(j \omega_{p}\right) C_{\gamma}\left(j \omega_{p}\right)=B_{p}$ and $G\left(j \omega_{g}\right) C_{\gamma}\left(j \omega_{g}\right)=B_{g}$. These relations can be rewritten as

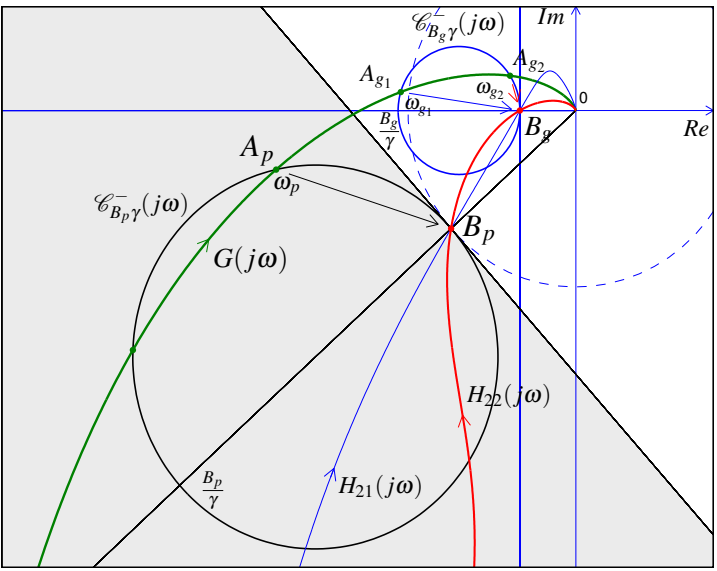

Fig. 7. Graphical solution of the DPB on the Nyquist plane.

$$
\left\{\begin{array}{l}
\left.G(j \omega)\right|_{\omega=\omega_{p}}=\frac{B_{p}}{C_{\gamma}\left(j \omega_{p}\right)}=\left.\mathscr{C}_{B_{p} \gamma}^{-}(j \omega)\right|_{\omega=\omega_{p}} \\
\left.G(j \omega)\right|_{\omega=\omega_{g}}=\frac{B_{g}}{C_{\gamma}\left(j \omega_{g}\right)}=\left.\mathscr{C}_{B_{g} \gamma}^{-}(j \omega)\right|_{\omega=\omega_{g}}
\end{array}\right.
$$

b) Graphical Solution on Nichols plane. The graphical construction described above for the Nyquist plane can be carried out also on the Nichols plane, see Fig. 6. The shapes of $\mathscr{C}_{B_{p} \gamma}^{-}(j \omega)$ and $\mathscr{C}_{B_{g} \gamma}^{-} \gamma(j \omega)$ on the Nichols plane are not circles, but the intersection points $A_{p 1}, A_{p 2}, A_{g 1}$ and $A_{g 2}$ with $G(j \omega)$ can still be determined. An advantage of working on the Nichols plane is that $\mathscr{C}_{B_{p} \gamma}^{-}(j \omega)$ and $\mathscr{C}_{B_{g} \gamma}^{-}(j \omega)$ have the same shape and the same dimension. In fact, these functions differ for just a constant, $\mathscr{C}_{B_{g} \gamma}^{-}(j \omega)=\left(B_{g} / B_{p}\right) \mathscr{C}_{B_{p} \gamma}^{-}(j \omega)$, and therefore they differ for just a translation on the Nichols plane.

\subsection{Solutions of other Design Problems.}

The value of $\gamma$ in the Design Problem A can also be chosen to satisfy other requirements. Let us consider, for example, the following Design Problems.

Design Problem B: $\left(\phi_{m}, G_{m}, \omega_{p}\right)$. Given design specifications on the phase margin $\phi_{m}$, gain margin $G_{m}$ and gain crossover frequency $\omega_{p}$, design a lead-lag compensator $C(s)$ such that the loop gain transfer function $C(j \omega) G(j \omega)$ passes through point $B_{p}=e^{j\left(\pi+\phi_{m}\right)}$ for $\omega=\omega_{p}$ and through point $B_{g}=-1 / G_{m}$.

Solution B. The design specifications completely define the points $A_{p}=G\left(j \omega_{p}\right), B_{p}=e^{j\left(\pi+\phi_{m}\right)}$ and $B_{g}=-1 / G_{m}=$ $M_{B_{g}} e^{j \varphi_{B_{g}}}$. The set $C_{p}\left(s, \omega_{g}\right)$ of all the lead-lag compensators $C(s)$ which solve Design Problem B (DPB) is obtained from (1) using the parameters

$$
\begin{gathered}
\gamma=\frac{X_{p}}{Y_{p}}>0, \quad \delta=Y_{p} \frac{\omega_{n}^{2}-\omega_{p}^{2}}{2 \omega_{n} \omega_{p}}>0 \\
\omega_{n}=\sqrt{\frac{Y_{g} \omega_{g}-Y_{p} \omega_{p}}{\frac{Y_{g}}{\omega_{g}}-\frac{Y_{p}}{\omega_{p}}}>0}
\end{gathered}
$$

where the coefficients $X_{p}=X\left(A_{p}, B_{p}\right), Y_{p}=Y\left(A_{p}, B_{p}\right), X_{g}=$ $X\left(A_{g}, B_{g}\right)$ and $Y_{g}=Y\left(A_{g}, B_{g}\right)$ are obtained using the inversion formulae (11) with $A_{g}=G\left(j \omega_{g}\right)=M_{A_{g}}\left(\omega_{g}\right) e^{j \varphi_{A_{g}}\left(\omega_{g}\right)}$, for all the frequencies $\omega_{g}$ satisfying the relation 


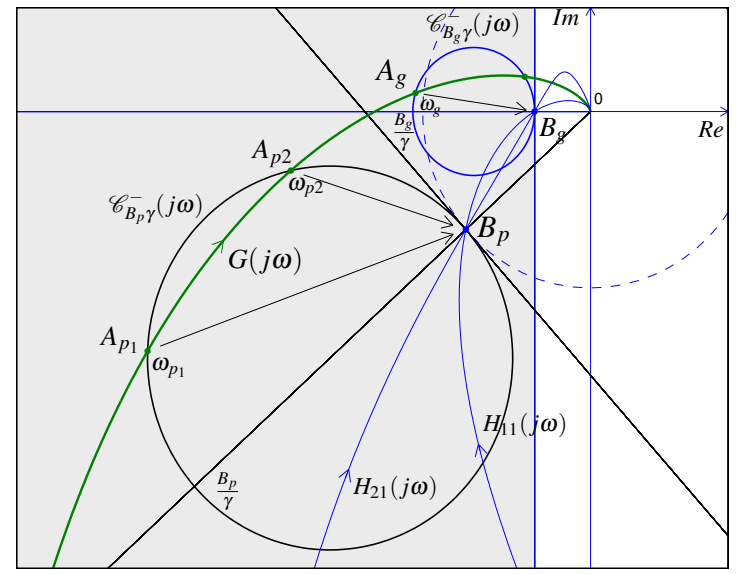

Fig. 8. Graphical solution of the DPC on the Nyquist plane.

$$
\gamma=\gamma_{g}\left(\omega_{g}\right)
$$

where $\gamma_{g}\left(\omega_{g}\right)=X_{g} / Y_{g}$ is defined as

$$
\gamma_{g}\left(\omega_{g}\right)=\frac{\frac{M_{B_{g}}}{M_{A_{g}}\left(\omega_{g}\right)}-\cos \left(\varphi_{B_{g}}-\varphi_{A_{g}}\left(\omega_{g}\right)\right)}{\cos \left(\varphi_{B_{g}}-\varphi_{A_{g}}\left(\omega_{g}\right)\right)-\frac{M_{A_{g}}\left(\omega_{g}\right)}{M_{B_{g}}}}
$$

A solution $C_{p}\left(s, \omega_{g}\right)$ of Design Problem B exists only if: 1) the set $S_{\omega_{g}}$ of all the $\omega_{g}$ satisfying (23) is not empty; 2) $A_{p} \in \mathscr{D}_{B_{p}}^{-}$ and $A_{g} \in \mathscr{D}_{B_{g}}^{-}$; 3) $\omega_{n}$ and $\delta$ in (21) and (22) are real and positive.

The graphical solution of the Design Problem B (DPB) on the Nyquist plane is shown in Fig. 7. The two loop gain frequency responses $H_{21}(s)$ (blue line) and $H_{22}(s)$ (red line) satisfy the gain margin $G_{m}$ at frequencies $\omega_{g 1}$ and $\omega_{g 2}$.

Design Problem C: $\left(\phi_{m}, G_{m}, \omega_{g}\right)$. Given design specifications on the phase margin $\phi_{m}$, gain margin $G_{m}$ and phase crossover frequency $\omega_{g}$, design a lead-lag compensator $C(s)$ such that the loop gain transfer function $C(j \omega) G(j \omega)$ passes through point $B_{g}=-1 / G_{m}$ for $\omega=\omega_{g}$ and through point $B_{p}=e^{j\left(\pi+\phi_{m}\right)}$.

Solution C. The design specifications completely define the points $A_{g}=G\left(j \omega_{g}\right), \quad B_{p}=e^{j\left(\pi+\phi_{m}\right)}$ and $B_{g}=-1 / G_{m}=$ $M_{B_{g}} e^{j \varphi_{B_{g}}}$. The set $C_{g}\left(s, \omega_{p}\right)$ of all the lead-lag compensators $C(s)$ which solve Design Problem $C$ is obtained from (1) using the parameters

$$
\begin{gathered}
\gamma=\frac{X_{g}}{Y_{g}}>0, \quad \delta=Y_{g} \frac{\omega_{n}^{2}-\omega_{g}^{2}}{2 \omega_{n} \omega_{g}}>0 \\
\omega_{n}=\sqrt{\frac{Y_{g} \omega_{g}-Y_{p} \omega_{p}}{\frac{Y_{g}}{\omega_{g}}-\frac{Y_{p}}{\omega_{p}}}>0}
\end{gathered}
$$

where the coefficients $X_{g}=X\left(A_{g}, B_{g}\right), Y_{g}=Y\left(A_{g}, B_{g}\right), X_{p}=$ $X\left(A_{p}, B_{p}\right)$ and $Y_{p}=Y\left(A_{p}, B_{p}\right)$ are obtained using the inversion formulae (11) with $A_{p}=G\left(j \omega_{p}\right)=M_{A_{p}}\left(\omega_{p}\right) e^{j \varphi_{A_{p}}\left(\omega_{p}\right)}$, for all the frequencies $\omega_{p}$ satisfying $\gamma=\gamma_{p}\left(\omega_{p}\right)$ where $\gamma_{p}\left(\omega_{p}\right)=$ $X_{p} / Y_{p}$ is defined as

$$
\gamma_{p}\left(\omega_{p}\right)=\frac{\frac{M_{B_{p}}}{M_{A_{p}}\left(\omega_{p}\right)}-\cos \left(\varphi_{B_{p}}-\varphi_{A_{p}}\left(\omega_{p}\right)\right)}{\cos \left(\varphi_{B_{p}}-\varphi_{A_{p}}\left(\omega_{p}\right)\right)-\frac{M_{A_{p}}\left(\omega_{p}\right)}{M_{B_{p}}}} .
$$

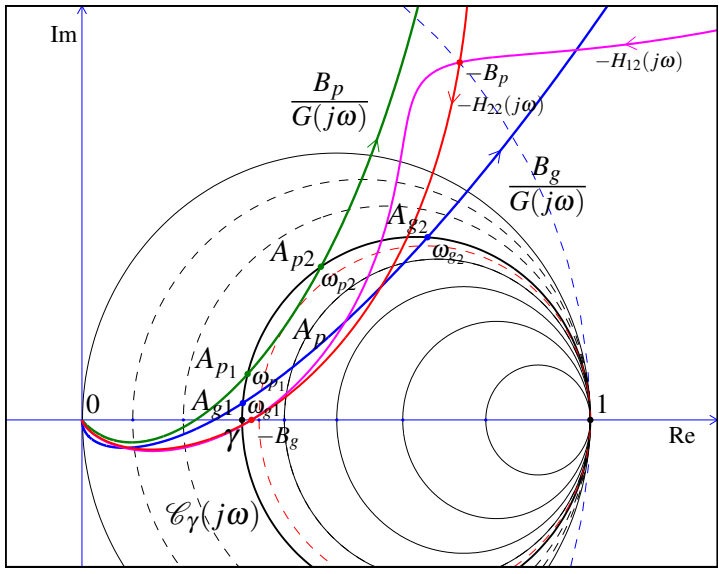

Fig. 9. $\mathscr{C}_{\gamma}(s)$-graphical representation on Nyquist plane.

A solution $C_{g}\left(s, \omega_{p}\right)$ of Design Problem $C$ exists only if: 1) the set $S_{\omega_{p}}$ of all the $\omega_{p}$ satisfying $\gamma=\gamma_{p}\left(\omega_{p}\right)$ is not empty; 2) $A_{g} \in \mathscr{D}_{B_{g}}^{-}$and $A_{p} \in \mathscr{D}_{B_{p}}^{-}$; 3) $\omega_{n}$ and $\delta$ in (26) are real and positive.

The graphical solution of the Design Problem C (DPC) on the Nyquist plane is shown in Fig. 8. The two loop gain frequency responses $H_{11}(s)$ and $H_{21}(s)$ (thin blue lines) satisfy the phase margin $\phi_{m}$ at frequencies $\omega_{p 1}$ and $\omega_{p 2}$.

\subsection{Other Graphical Representations.}

The graphical solutions on Nyquist and Nichols planes shown in Fig. 5, Fig. 6, Fig. 7 and Fig. 8 can also be performed graphically in other ways. The following two cases are considered.

1) $\mathscr{C}_{\gamma}(s)$-graphical representation on Nyquist plane. Using simple mathematical manipulations, system (20) can be rewritten as:

$$
\left\{\begin{array}{l}
\left.\frac{B_{p}}{G(j \omega)}\right|_{\omega=\omega_{p}}=\left.\mathscr{C}_{\gamma}(j \omega)\right|_{\omega=\omega_{p}} \\
\left.\frac{B_{g}}{G(j \omega)}\right|_{\omega=\omega_{p}}=\left.\mathscr{C}_{\gamma}(j \omega)\right|_{\omega=\omega_{g}}
\end{array}\right.
$$

The graphical representation of (28) on the Nyquist plane is shown in Fig. 9: the intersections of $\mathscr{C}_{\gamma}(j \omega)$ with $B_{p} / G(j \omega)$ provide the frequencies $\omega_{p 1}$ and $\omega_{p 2}$, while the intersections of $\mathscr{C}_{\gamma}(j \omega)$ with $B_{g} / G(j \omega)$ provide the frequencies $\omega_{g 1}$ and $\omega_{g 2}$. In Fig. 9 the points $-B_{p}$ and $-B_{g}$, and the two loop gain frequency responses $-H_{12}(s)$ and $-H_{22}(s)$ are also reported: the relative position of these functions with respect to point 1 is the same of functions $H_{12}(s)$ and $H_{22}(s)$ with respect to point -1 .

2) $\mathscr{C}_{\gamma}(s)$-graphical representation on Nichols plane. The graphical representation of relations (28) can also be done on the Nichols plane as shown in Fig. 10. In this case the shapes of $\mathscr{C}_{\gamma}(j \omega)$ are not circles, but the graphical representation is more precise for small values of the modulus. This graphical representation is quite similar to the one presented by Yeung et al. (1998) for the solution of Design Problems B and C.

\section{NUMERICAL EXAMPLES}

Let us consider the transfer function, see Yeung et al. (1998):

$$
G(s)=\frac{5000}{s(s+5)(s+10)},
$$




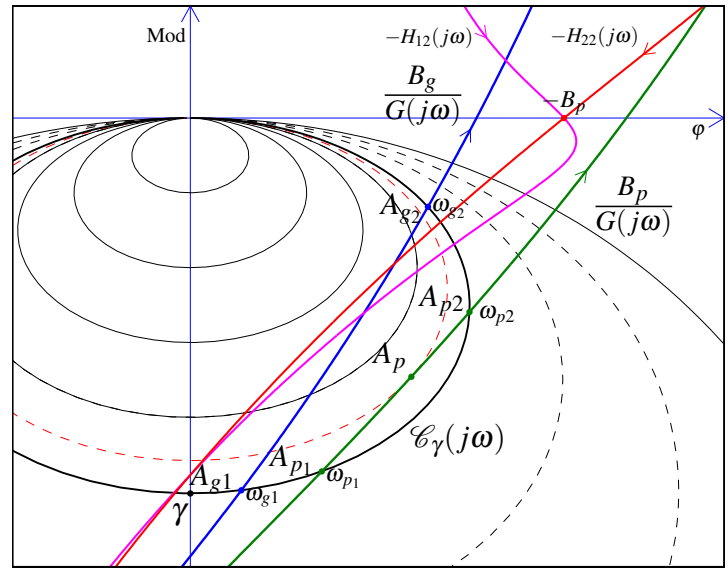

Fig. 10. $\mathscr{C}_{\gamma}(s)$-graphical representation on Nichols plane.

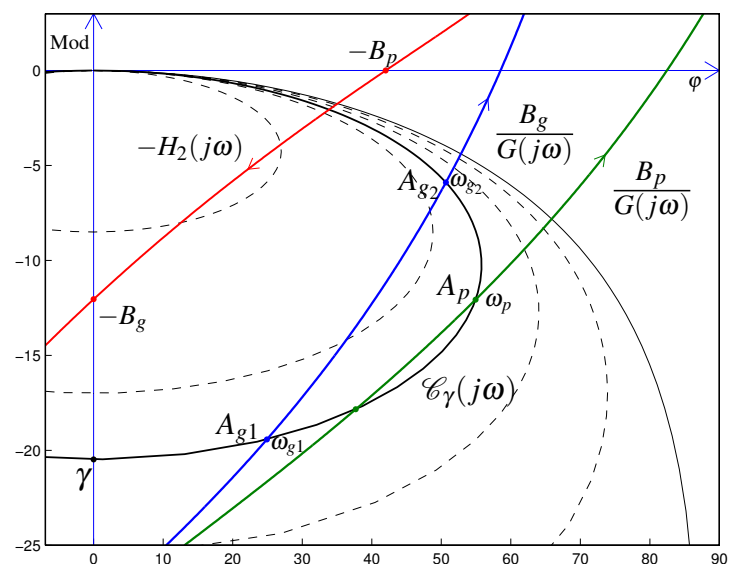

Fig. 11. Graphical solution of the numerical example on the Nichols plane.

with the following design specifications: phase margin $\phi_{m}=42^{\circ}$, gain crossover frequency $\omega_{p}=9 \mathrm{rad} / \mathrm{s}$ and gain margin $G_{m}=4$.

The synthesis of the lead-lag controllers $C_{p}\left(s, \omega_{g 2}\right)$ follows the line described in the Design Problem B. The design specifications define points $B_{p}=e^{j 222^{\circ}}, A_{p}=G\left(j \omega_{p}\right)=4.01 e^{j 167.1^{\circ}}$ and $B_{g}=0.25 e^{j 180^{\circ}}$. From points $A_{p}$ and $B_{p}$ one obtains $X_{p}=$ $-0.397, Y_{p}=-4.198$ and $\gamma=X_{p} / Y_{p}=0.0946$. The two solutions of equation (23) are $S_{\omega_{g}}=\left\{\omega_{g 1}, \omega_{g 2}\right\}=\{11.37,20.71\}$. Only the solution corresponding to frequency $\omega_{g 2}$ is admissible: $\delta=17.68$ and $\omega_{n}=2.28$. The corresponding lead-lag compensator is:

$$
C_{p}\left(s, \omega_{g 2}\right)=\frac{s^{2}+3.347 s+5.198}{s^{2}+35.36 s+5.198} .
$$

The graphical solutions on the Nichols and Nyquist planes corresponding to the synthesis of the lead-lag compensator $C_{p}\left(s, \omega_{g 2}\right)$ are shown in Fig. 11 and Fig. 12, respectively. The red line plotted in the two figures corresponds to the loop gain transfer function $H_{2}(j \omega)=C_{p}\left(j \omega, \omega_{g 2}\right) G(j \omega)$.

Final Remark. The graphical solution of the Design Problems B and C for a lead-lag compensator with real poles and zeros was given by Yeung et al. (1998) using a "design chart" similar to the graphical construction shown in Fig. 11. The main differences with the solution proposed in this paper are the following: 1) in Yeung et al. (1998) the parameters $T_{1}, T_{2}$ and $\beta$ of the lead-lag compensator are graphically determined using the "design chart" and the intermediate parameters $\tau, \Omega_{1}$ and $\Omega_{2}$;

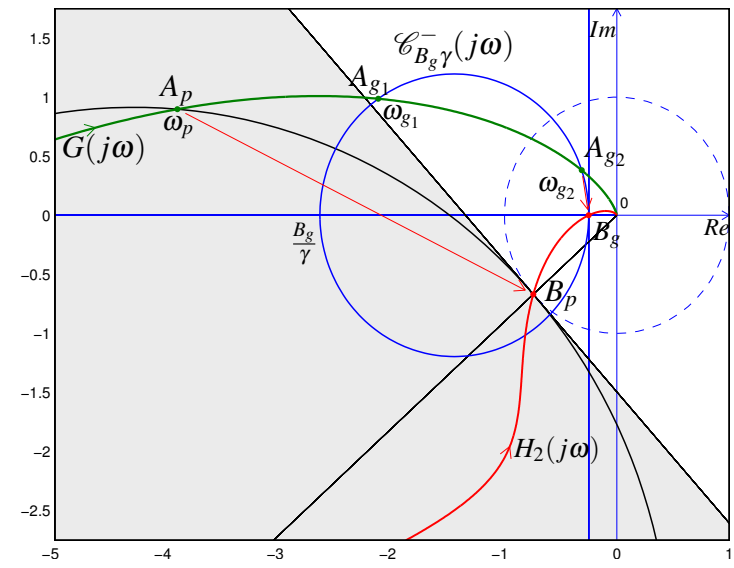

Fig. 12. Graphical solution of the numerical example on the Nyquist plane.

In our method the compensator's parameters $\gamma, \delta$ and $\omega_{n}$ are exactly determined using simple formulae. 2) In both methods the set $S_{\omega g}$ is determined in similar way, but in Yeung's method only one of the two admissible solutions of (23) is determined; 3) No constraints are introduced in Yeung et al. (1998) on the parameters $T_{1}, T_{2}$ and $\beta$, so the obtained compensator can also be unstable; 4) The design method presented in this paper has a nice graphical interpretation on the Nyquist plane which is not discussed in Yeung et al. (1998).

\section{REFERENCES}

Flores S.S., A.M. Valle and B.A. Castillejos Geometric Design of Lead/Lag Compensators Meeting a Hinf Specification. ICEEE Conference, Mexico City, Mexico, September 5-7, 2007.

Fung H.-W., Wang Q.-G. and Lee T.-H., PI tuning in terms of gain and phase margins. Automatica: 34(9):1145-9,1998.

Ho W.K., C.C. Hang and L.S. Cao. Tuning of PID controllers based on gain and phase margin specifications. Automatica: 31(3):497-502, 1995.

Marro G. and R. Zanasi, New Formulae and Graphics for Compensator Design. IEEE International Conference On Control Applications, Trieste, Italy, September 1-4, 1998.

Messner W.C., M.D. Bedillion, L. Xia and D.C. Karns. Lead and Lag Compensators with Complex Poles and Zeros: design formulas for modeling and loop shaping. IEEE Control Systems Magazine, vol. 27, no. 1, pp.44-54, Feb 2007.

Messner W. Formulas for Asymmetric Lead and Lag Compensators. American Control Conference, Hyatt Regency Riverfront, St. Louis, MO, USA, June 10-12, 2009.

Phillips C.L. Analytical Bode Design of Controllers. IEEE Transactions on Education, E-28, no. 1, pp. 43-44, 1985.

Wang Q.-G., Fung H.-W., Zhang Y., PID tuning with exact gain and phase margins. ISA Transactions, 38,243-249, 1999.

Yeung K.S., K.W. Wong and K.L. Chen, A Non-Trial-andError Method for Lag-Lead Compensator Design. IEEE Transactions on Education, E-41, no. 1, February 1998.

Zanasi R., R. Morselli, Discrete Inversion Formulas for the Design of Lead and Lag Discrete Compensators. ECC - European Control Conference, 23-26 August 2009, Budapest, Hungary.

Lee C.H. A survey of PID controller design based on gain and phase margins. International Journal of Computational Cognition: 2(3):63-100, 2004. 